\title{
ORIGINAL
}

\section{CLASIFICACIÓN DE LAS PERSONAS DEPENDIENTES A PARTIR DE LA ENCUESTA DE DISCAPACIDADES, DEFICIENCIAS Y ESTADO DE SALUD DE 1999 (*)}

\section{Irene Albarrán Lozano y Pablo Alonso González}

Universidad de Extremadura. Facultad de Estudios Empresariales y Turismo.

(*) Este trabajo ha contado con la financiación del Ministerio de Educación y Ciencia y el Plan Nacional de Investigación Científica, Desarrollo e InnovaciónTecnológica a través del proyecto SEJ2005-08070/ECON, cofinanciado con fondos FEDER

\section{RESUMEN}

Fundamento: En relación con las personas en situación de dependencia, para pasar a un sistema en el que prime el cuidado formal médico social, en el que tenga más peso el papel de las instituciones, conviene analizar las distintas realidades que presentan estas personas. El objetivo de este trabajo consiste en la clasificación de la población española en situación de dependencia en distintos grupos, cada uno de ellos con unas características diferenciadas.

Métodos: Se analizan los datos de la Encuesta de Discapacidades, Deficiencias y Estado de Salud (INE, 1999) referidos a la población mayor de 6 años que no está institucionalizada. Mediante la utilización de técnicas de análisis multivariante se configuran grupos en función de la edad, el sexo, las discapacidades relacionadas con las actividades de la vida diaria, la severidad de las mismas y el número de horas de cuidados por semana.

Resultados: Se obtienen nueve perfiles estadísticamente heterogéneos. En los tres primeros, con 341.262 personas $(24,5 \%$ de la población total dependiente), se tiene menos de 60 años. Salvo en un grupo predominan las mujeres (61\% varones) con edades entre 13 y 36 años. En el grupo menos afectado por la falta de autonomía (136.240 personas) presentan problemas para desplazarse sólo el $26 \%$ de sus integrantes (en severidad máxima el $7 \%$ ), de cuidado el $25 \%$ ( $10 \%$ en severidad máxima) y sólo el $32 \%$ necesitan más de 15 horas semanales de cuidado. Son, en su mayoría (74\%), mujeres de entre 82 a 90 años.

Conclusiones: La población dependiente española presenta distintas realidades que responden a diversas categorías en función de la edad, el sexo, el número y severidad de las actividades de la vida diaria afectadas. Las distintas valoraciones de la severidad asociada a dichas categorías, utilizando tanto el criterio de máxima severidad del INE como otro criterio alternativo que introduce el nivel de severidad de todas las actividades de la vida diaria no sólo de la máxima, confirman la distinción de los nueve grupos encontrados.

Palabras clave: Cuidados a largo plazo. Actividades de la vida diaria. Análisis por conglomerados.

Correspondencia:

Irene Albarrán Lozano

Facultad de Estudios Empresariales y Turismo

Universidad de Extremadura

Avda. Universidad s/n

10071 Cáceres

Correo electrónico: ialbaloz@unex.es
ABSTRACT

\section{Dependent Individuals Classification Based on the 1999 Disabilities, Impairments and Health Status Survey}

Background: In order to move to a system in which formal medical-social care is a priority, in which the institutions play a greater role, it is advisable to analyze the different actual situations of those individuals who are dependent. The objective of this study consists of classifying the dependent Spanish population into different groups, each of their own distinguishing characteristics.

Methods: The data from the Disabilities, Deficiencies and Health Status Survey (Spanish National Institute of Statistics, 1999) related to the non-institionalized population over 6 years of age. Groups are configured using multivariate analysis techniques in terms of the age, sex, daily living activity-related disabilities, the severity thereof and the number of hours of care per week.

Results: Nine statistically heterogeneous profiles were obtained. In the first three, with 341,262 individuals $(24.5 \%$ of the total dependent population), the individuals in question were under 60 years of age. With the exception of one group, females were predominant (61\% males), falling within the $13-36$ age range. In the group least affected by lack of self-sufficiency (136,240 individuals), solely $26 \%$ of those comprising this group had problems getting around (maximum degree of severity in $7 \%$ ), regarding care, $25 \%$ (maximum degree of severity in 10\%) and only $32 \%$ need more than 15 hours of care per week. Most thereof $(74 \%)$ are females within the 82-90 age range.

Conclusions: The dependent Spanish population is revealed to be in different situations related to different categories in terms of age, sex, number and severity of the daily living activities affected. The different assessments of the severity related to said categories, employing both the Spanish National Institute of Statistics maximum severity criterion as well as another alternative criterion which implements the degree of severity of all of the daily living activities, not only the maximum, confirm the nine groups found to be different from one another. ring

Key words: Long-term care. Activities of daily living. Cluste- 


\section{INTRODUCCIÓN}

Hasta ahora la atención a las personas dependientes ha recaído en la familia y, por tanto, han prevalecido los llamados cuidados informales. Si lo que se pretende es pasar de este sistema a otro en el que primen los cuidados formales médicosociales ${ }^{1}$ y tenga más peso el papel de las instituciones conviene analizar primeramente las distintas realidades que presentan las personas en situación de dependencia.

En este trabajo se pretende profundizar en los perfiles específicos que pueden presentar las personas dependientes, centrándose en la simultaneidad de diferentes discapacidades -la denominada codiscapacidad-, y en su severidad, para así tener una idea más clara de estas situaciones y poder enfocar sus soluciones con planteamientos más adecuados a la realidad. Se considera importante ahondar en la idea de codiscapacidad ${ }^{1}$ vinculándola a la ayuda para realizar las actividades cotidianas y su dimensión social ${ }^{2,3}$.

Dado que este trabajo se centra en las personas dependientes, parece oportuno delimitar exactamente qué se entiende por tal. No existe una definición única y homogénea respecto al fenómeno de la dependencia. $\mathrm{La}$ mayoría de las acepciones se reducen o centran en los aspectos físicos o mentales añadiéndose, en ocasiones, la dimensión social y económica ${ }^{4}$. Así, la dependencia puede definirse desde cuatro ejes: aspectos físicos (basados en escalas como la de Katz), aspectos mentales, dimensión social y dimensión económica ${ }^{5}$.

Para este trabajo nos centraremos en la definición de la dependencia proporcionada por el Consejo de Europa ${ }^{6}$, según la cual es el estado en el que se encuentra una persona que necesita la asistencia de otra para realizar determinadas actividades. De esta forma, no se asocia la situación de dependencia a personas con edades superiores a 64 años.
Respecto a la búsqueda y definición de patrones de comportamiento existen investigaciones que en la mayoría de las ocasiones buscan una tipología de usuarios o demandantes de determinados servicios sanitarios, cuidados formales e informales ${ }^{7-9}$. En otras ocasiones centran el estudio en algún tipo específico de enfermedad o discapacidad ${ }^{10}$. Lo más frecuente es focalizar los estudios en las dimensiones física y mental, principalmente en la primera, utilizando el término de capacidad funcional $^{11}$.

En la definición de qué actividades cotidianas están asociadas a la dependencia, la mayoría de los trabajos desde los orígenes de los estudios se centran en las actividades de la vida diaria $(A V D)^{12,13}$. Para este estudio se utilizan las enumeradas por el INE en la Encuesta de Discapacidades, Deficiencias y Estado de Salud (EDDES) de 1999, que son las siguientes: 1) realizar cambios de las diversas posiciones del cuerpo y mantenerse, 2) levantarse, acostarse, permanecer de pie o sentado, 3) desplazarse dentro del hogar, 4) deambular sin medio de transporte, 5) asearse solo, 6) controlar las necesidades e ir solo al servicio, 7) vestirse, desvestirse y arreglarse, 8) comer y beber, 9) encargare de las compras, 10) encargarse de las comidas, 11) encargarse de la limpieza y el planchado de la ropa, 12) encargarse de la limpieza y mantenimiento de la casa, 13) encargarse del bienestar de la familia. Las ocho primeras son denominadas actividades básicas de la vida diaria -ABVD- y las restantes actividades instrumentales de la vida diaria -AIVD-.

En concreto, para clasificar a las personas en situación de dependencia existen varios criterios ${ }^{14}$ basados en considerar bien el número y naturaleza de las AVD bien las diversas escalas de capacidad funcional muy utilizadas en el ámbito clínico-sanitario (como los índices de Katz, Lawton y Brody, por ejemplo) o bien en la utilización de técnicas multivariantes. 
En este artículo se parte del tercero de ellos, puesto que la utilización de técnicas multivariantes no presupone ninguna hipótesis de partida sobre el número de categorías de la dependencia o el contenido de las mismas. Del mismo modo que Zunzunegui y Béland $^{11}$, en este análisis se introducen variables de diversa naturaleza, no sólo relacionadas con la dificultad o no para realizar las AVD (tal y como otros autores consideraban ${ }^{15-17}$ ) sino también el grado de severidad y otras como el sexo, la edad o el estado civil. Esta investigación amplía el estudio de Zunzunegui y Béland ${ }^{11}$ puesto que, partiendo de una preselección de la población en situación de dependencia, tal y como se ha definido previamente, se recurre a la utilización de técnicas de segmentación para ver qué perfiles o realidades describen.

\section{MATERIAL Y MÉTODOS}

\section{Población de estudio:}

El estudio se centra en la población de más de seis años reflejada en la EDDES que requiera la ayuda de otra persona. Esta encuesta fue elabora por el INE en colaboración con el IMSERSO y la Fundación ONCE durante 1999 y recoge información sobre 70.500 hogares y unas 220.000 personas en toda España. Es decir, se recogía información referida únicamente a personas que residen en vivienda familiar. Aplicando a cada persona encuestada su factor de elevación en función del muestreo estadístico realizado por el INE, se garantiza la representatividad nacional de la encuesta.

La EDDES adoptó la Clasificación Internacional de Deficiencias, Discapacidades y Minusvalías propuesta por la Organización Mundial de la Salud en 1980 y posteriormente fue modificada ${ }^{18}$, identificando 36 posibles discapacidades agrupadas en 10 bloques. A su vez, para cada una de las discapacidades se recoge el grado de severidad con que la padece el individuo, distinguién- dose entre moderada, severa y total, existiendo, obviamente, un cuarto nivel asociado a la inexistencia de la discapacidad. Dado que el fenómeno de la dependencia toma carácter definitivo al incorporar la ayuda de otra persona, eso sugiere que puede ocurrir a cualquier edad ${ }^{19}$. Por ello, el estudio no se ha restringido a las personas de más de 64 años, sino a toda la población no institucionalizada mayor de seis años, puesto que antes de esta edad no se habla de discapacidades sino de limitaciones ${ }^{20}$.

\section{Medida de las variables}

Las variables del estudio son: la edad (en años), variables dicotómicas que recogen la existencia o no de una característica como son las asociadas a las discapacidades, la presencia de discapacidades asociadas a las ABVD y la posesión o no de certificado de minusvalía (1 es sí, 0 es no), el sexo del encuestado ( 1 es hombre, 0 es mujer), las horas semanales de cuidados siguiendo la codificación del INE (1 para menos 7 horas, 2 entre 7 y 14 horas, 3 entre 15 y 30 horas, 4 entre 31 y 40 horas, 5 entre 41 y 60 horas y 6 para más de 60 horas) y la severidad de la discapacidad, también siguiendo la codificación del $\mathrm{INE}^{20}$ ( 1 en el caso de sin dificultad, 2 dificultad moderada, 3 dificultad grave y 4 imposibilidad de realizar la actividad).

\section{Análisis estadístico}

Tras la selección de las personas participantes según el criterio establecido, el siguiente eje del trabajo consistió en tener en cuenta el grado de dependencia de la población afectada para proceder a su clasificación. Para ello se utilizaron técnicas multivariantes para clasificar a la población dependiente española en grupos, buscando la máxima homogeneidad interna y heterogeneidad externa (mayor igualdad entre los individuos que integren un grupo y diferenciación con el resto). Finalmente, se com- 
probó que los perfiles encontrados responden a situaciones distintas contrastadas estadísticamente.

La EDDES facilita información sobre el número de horas semanales que una persona destina al cuidado de un individuo con discapacidad, si bien la codifica en intervalos. La definición de persona dependiente es la utilizada por Puga ${ }^{19}$, entendiendo por dependencia «el hecho de no poder valerse por sí mismo en algún grado, es decir, tener dificultades o no poder realizar determinadas actividades habituales para el conjunto de la población». O sea, se requiere ayuda para la realización de una o varias de estas actividades o, sencillamente, no pueden realizarse.

Para llevar a cabo la investigación se han seguido los siguientes pasos:

\section{Selección de la población dependiente}

Se consideró como criterio de selección la definición del Consejo de Europa en sentido amplio, es decir, padecer cualquiera de las trece AVD y tener registradas horas de cuidado. De esta forma no se han considerado otros criterios más restrictivos ampliamente utilizados, como el índice de Katz ${ }^{12}$, que sólo considera seis de las AVD, el índice de Barthel $^{21}$, que sólo distingue dos grados de severidad, o el índice de Lawton y Brody ${ }^{13}$, que detalla actividades que no han sido consideradas en la encuesta, como por ejemplo, la utilización del teléfono.

\section{División en grupos heterogéneos de la población analizada}

Partiendo de la hipótesis de que las discapacidades que afectan a las 13 AVD no se presentan por igual en todos los individuos, será aceptada si del conjunto de variables que caracterizan a cada individuo se obtienen, mediante el análisis cluster, grupos diferentes estadísticamente significativos. En primer lugar se realizaron técnicas de análisis jerárquico y, una vez decidido el número de grupos a tener en cuenta, técnicas de análisis no jerárquico (o de $k$ medias) probando con distinto número de grupos. Se emplea como medida de distancia la euclídea al cuadrado:

$$
d_{i j}^{2}=\sum_{k=1}^{p}\left(X_{i k}-X_{j k}\right)^{2}
$$

donde: $\boldsymbol{d}_{i j}^{2}$ representa la distancia entre los individuos $i$ y $j, X_{i k}$ es el valor de la variable $X_{k}$ para el individuo $i X_{j k}$ y el valor de la variable para el individuo $j$.

Tras probar con los distintos métodos de agrupamiento posibles elegido fue el de Ward, que minimiza la variación interna del grupo obtenido. Tras analizar el dendrograma se realizaron varias pruebas utilizando el algoritmo $k$ medias para finalmente seleccionar la clasificación correspondiente a nueve agrupaciones.

Se contrastó estadísticamente la asociación dos a dos de las distintas discapacidades mediante el test Chi-cuadrado ${ }^{22}$ cuyos resultados aparecen en la tabla 1 . Se rechaza en todos los casos al 5\% la no relación, agrupándose las discapacidades por bloques: DESPLAZARSE (discapacidades 1 a 3), DEAMBULAR (únicamente la discapacidad 4), CUIDARSE (discapacidades 5 a la 8) y TAREAS (discapacidades 9 a 13). Las variables agrupadas presentan el valor 1 si el individuo registra cualquiera de las discapacidades agrupadas y 0 en caso contrario. Tras varias pruebas y técnicas multivariantes de segmentación se confirmó un mayor poder diferenciador agrupando por bloques que cada discapacidad por separado. Como severidad del bloque se asoció la máxima registrada en cualquiera de las discapacidades integrantes siguiendo el criterio del INE ${ }^{23}$.

\section{Análisis y comparación de los grupos obtenidos}

Una vez obtenidos los grupos o categorías de dependencia, se analizaron detalladamen- 
Tabla 1

Pruebas Chi-cuadrado por grupos de discapacidades

\begin{tabular}{|c|c|c|c|c|c|c|}
\hline \multicolumn{6}{|c|}{ DESPLAZARSE } & \\
\hline \multirow{2}{*}{\multicolumn{2}{|c|}{\begin{tabular}{|c|} 
DISCAPACIDADES \\
1
\end{tabular}}} & 1 & 2 & & 3 & \\
\hline & & -- & $\begin{array}{r}6170 \\
(0,0 \\
\end{array}$ & $\begin{array}{l}8,67 \\
00)\end{array}$ & \begin{tabular}{|c|}
442311,29 \\
$(0,000)$
\end{tabular} & \\
\hline \multicolumn{2}{|c|}{2} & & - & & \begin{tabular}{|c|}
588418,14 \\
$(0,000)$ \\
\end{tabular} & \\
\hline \multicolumn{2}{|c|}{3} & & & & -- & \\
\hline \multicolumn{7}{|c|}{ CUIDARSE } \\
\hline \multicolumn{2}{|c|}{\begin{tabular}{|l|} 
DISCAPACIDADES \\
\end{tabular}} & - & & 7 & 7 & 8 \\
\hline \multicolumn{2}{|l|}{5} & $\begin{array}{r}4341 \\
(0,1 \\
\end{array}$ & $\begin{array}{l}20,50 \\
000) \\
00\end{array}$ & $\begin{array}{r}7187 \\
0,0 \\
\end{array}$ & \begin{tabular}{l|r}
89,59 & 2628 \\
$000)$ & 0, \\
\end{tabular} & $\begin{array}{l}24,15 \\
000)\end{array}$ \\
\hline \multicolumn{2}{|l|}{6} & 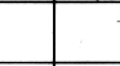 & 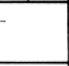 & $\begin{array}{r}5190 \\
(0,0 \\
\end{array}$ & \begin{tabular}{l|r}
95,32 & 537 \\
$000)$ & 0, \\
\end{tabular} & $\begin{array}{l}66,51 \\
000)\end{array}$ \\
\hline 7 & & & & - & $\begin{array}{r}3438 \\
\quad 0 . \\
\end{array}$ & $\begin{array}{l}96,67 \\
000)\end{array}$ \\
\hline \multicolumn{2}{|l|}{8} & & & & & - \\
\hline \multicolumn{7}{|c|}{ TAREAS } \\
\hline DISCAPACIDADES & 9 & \multirow{2}{*}{\begin{tabular}{|c|}
10 \\
387646,87 \\
$(0,000)$ \\
\end{tabular}} & \multicolumn{2}{|c|}{11} & 12 & 13 \\
\hline \begin{tabular}{|c|}
9 \\
9
\end{tabular} & - & & \multicolumn{2}{|c|}{$\begin{array}{c}366536,15 \\
(0,000) \\
\end{array}$} & $\begin{array}{c}285314,87 \\
(0,000) \\
\end{array}$ & $\begin{array}{c}332319,17 \\
(0,000)\end{array}$ \\
\hline 10 & & -- & \multicolumn{2}{|c|}{$\begin{array}{c}781794,27 \\
(0,000) \\
\end{array}$} & $\begin{array}{c}458617,65 \\
(0,000)\end{array}$ & $\begin{array}{c}646165,15 \\
(0,000)\end{array}$ \\
\hline 11 & & & \multicolumn{2}{|c|}{--} & $\begin{array}{c}774240,04 \\
(0,000) \\
\end{array}$ & $\begin{array}{c}517341,51 \\
(0,000)\end{array}$ \\
\hline 12 & & & & & -- & $\begin{array}{c}342817,11 \\
(0,000) \\
\end{array}$ \\
\hline 13 & & & & & & - \\
\hline \multicolumn{7}{|c|}{$\begin{array}{c}\text { Contraste } \chi^{2} \text { con } 1 \text { grado de libertad } \\
\text { Valor del estadístico } \\
\text { (significación) }\end{array}$} \\
\hline
\end{tabular}

te y se contrastaron estadísticamente mediante técnicas de análisis de la varianza, ANOVA, si existían diferencias significativas entre ellos para todas las variables que han intervenido en su formación. Para ello, se contrastó la hipótesis nula de igualdad entre todas las medias de cada uno de los grupos, es decir:

$$
\begin{aligned}
& H_{0}: \bar{x}_{1}=\bar{x}_{2}=\cdots=\bar{x}_{9}=\bar{x} \\
& H_{1}: \exists \bar{x}_{i} / \bar{x}_{i} \neq \bar{x} \quad i=1,2, \cdots, 9
\end{aligned}
$$

Si se aceptaba la hipótesis de diferencia de comportamiento, considerábamos que representaban perfiles diferenciados.

\section{Estimación del grado de severidad de la dependencia y comparación entre los grupos obtenidos}

Una vez determinados los grupos estadísticamente diferenciados, se procedió a cal- 
cular el valor que en cada uno de ellos alcanzaban tanto el índice propuesto por el INE como con el índice alternativo establecido por Alegre y otros investigadores ${ }^{24}$. Se contrastó y constató estadísticamente que cada categoría presentaba distinto grado de dependencia medido por los distintos valores de los índices utilizados.

\section{RESULTADOS}

Los análisis realizados sugieren la formación de nueve clusters o categorías diferentes, con un volumen de población distinto, tal y como se recoge en la tabla 2 . La tabla 3 recoge los resultados del test ANOVA, el cual indica que todas las variables introducidas en la formación de grupos resultan estadísticamente significativas al 5\%, evidenciando la existencia de grupos que reflejan situaciones distintas. La tabla 4 muestra los valores centrales (valores medios) para las distintas variables en los nueve grupos. Se aprecia, tal como confirma el test ANOVA de la tabla 3 , la heterogeneidad de los perfiles. Al calcular el valor de cada índice usando los valores medios de cada cluster (y a continuación evaluar el test $F$ de igualdad de medias con cada uno de los índices) las cifras que se obtienen son 39.255,3 para el caso del índice INE y $11.680,84$ para el caso del índice alternativo ${ }^{24}$. En ambos casos, los grados de libertad son 8 para el numerador $y$ 1.394.912 para el denominador, con lo que el valor de $\mathrm{p}$ en cada uno de ellos es prácticamente cero, rechazándose la hipótesis de igualdad conjunta de medias en los dos casos. Los valores de los dos índices para los centros de cada uno de los conglomerados o categorías de dependencia se recogen en la figura 1.

El grupo de población dependiente seleccionado consta de 1.394 .921 personas que cumplen las condiciones antes señaladas.

Tabla 2

Volumen de población en cada categoría de dependencia

\begin{tabular}{|c|c|c|}
\hline Cluster & No ponderado & Ponderado \\
\hline 1 & 632 & 119.246 \\
\hline 2 & 111 & 18.774 \\
\hline 3 & 1.196 & 203.242 \\
\hline 4 & 1.955 & 322.516 \\
\hline 5 & 1.144 & 198.170 \\
\hline 6 & 1.115 & 188.495 \\
\hline 7 & 807 & 136.240 \\
\hline 8 & 728 & 121.297 \\
\hline 9 & 542 & 86.941 \\
\hline Número de personas & 8.230 & 1.394 .921 \\
\hline Datos no válidos & 22 & 3.846 \\
\hline
\end{tabular}


Tabla 3

ANOVA

\begin{tabular}{|c|c|c|c|c|c|c|}
\hline \multirow[b]{2}{*}{ VARIABLES } & \multicolumn{2}{|c|}{ CONGLOMERADO } & \multicolumn{2}{|c|}{ ERROR } & \multirow[b]{2}{*}{$\mathbf{F}$} & \multirow[b]{2}{*}{ Signif. } \\
\hline & $\begin{array}{c}\text { MEDIA } \\
\text { CUADRÁTICA }\end{array}$ & g 1 & $\begin{array}{c}\text { MEDIA } \\
\text { CUADRÁTICA }\end{array}$ & gl & & \\
\hline EDAD & $61.480 .274,562$ & 8 & 35,524 & 1.394 .912 & $1.730 .676,001$ & 0,000 \\
\hline ABVD & 590,132 & 8 & 0,126 & 1.394 .912 & $4.678,951$ & 0,000 \\
\hline ESTADO CIVIL & $28.545,648$ & 8 & 0,439 & 1.394 .912 & $64.973,988$ & 0,000 \\
\hline CERTIF. MINUSVALÍA & $6.572,431$ & 8 & 0,146 & 1.394 .912 & $44.935,468$ & 0,000 \\
\hline HORAS SEMANALES & $16.968,555$ & 8 & 3,638 & 1.394 .912 & $4.664,816$ & 0,000 \\
\hline SEXO & $1.632,122$ & 8 & 0,212 & 1.394 .912 & $7.700,506$ & 0,000 \\
\hline \multicolumn{7}{|l|}{ DISCAPACIDADES: } \\
\hline DESPLAZARSE & 728,372 & 8 & 0,245 & 1.394 .912 & $2.970,456$ & 0,000 \\
\hline DEAMBULAR & $1.515,594$ & 8 & 0,193 & 1.394 .912 & $7.866,417$ & 0,000 \\
\hline CUIDARSE & $1.593,046$ & 8 & 0,240 & 1.394 .912 & $6.627,237$ & 0,000 \\
\hline TAREAS & 329,780 & 8 & 0,131 & 1.394 .912 & $2.517,566$ & 0,000 \\
\hline \multicolumn{7}{|l|}{ SEVERIDADES: } \\
\hline DESPLAZARSE & $5.882,042$ & 8 & 2,097 & 1.394 .912 & $2.804,854$ & 0,000 \\
\hline DEAMBULAR & $17.649,791$ & 8 & 1,846 & 1.394 .912 & $9.563,485$ & 0,000 \\
\hline CUIDARSE & $14.998,366$ & 8 & 2,231 & 1.394 .912 & $6.721,326$ & 0,000 \\
\hline TAREAS & $9.159,235$ & 8 & 1,928 & 1.394 .912 & $4.749,566$ & 0,000 \\
\hline
\end{tabular}

$\mathrm{gl}=$ grados de libertad.

Signif. = significación estadística.

Figura 1

Valores centrales de cada cluster

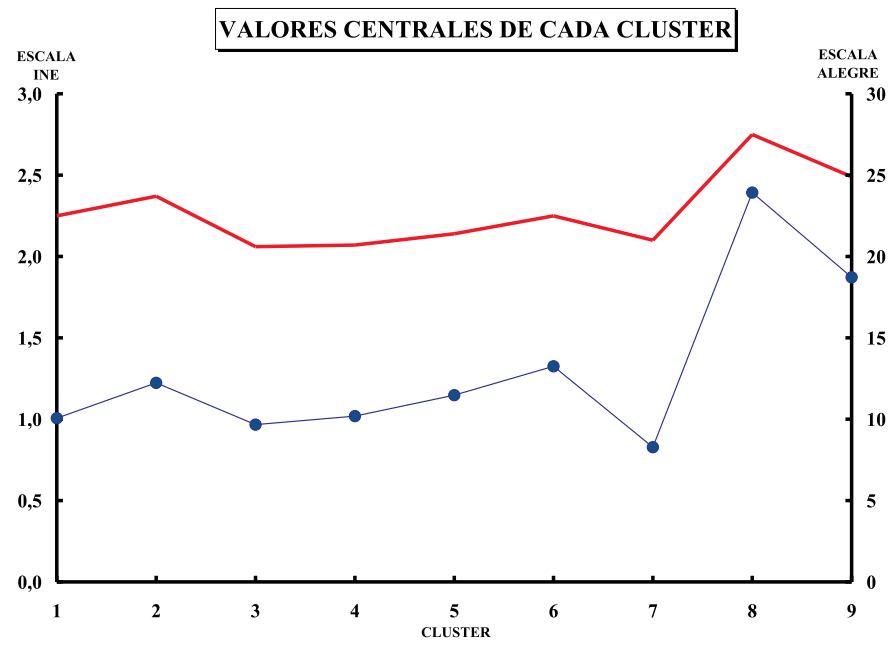


Tabla 4

Centros de los conglomerados finales

\begin{tabular}{|c|c|c|c|c|c|c|c|c|c|}
\hline \multicolumn{10}{|c|}{ CONGLOMERADO } \\
\hline & 1 & 2 & 3 & 4 & 5 & 6 & 7 & 8 & 9 \\
\hline EDAD & 9 & 26 & 50 & 67 & 75 & 80 & 85 & 86 & 93 \\
\hline $\mathrm{ABVD}$ & 1 & 1 & 1 & 1 & 1 & 1 & 1 & 1 & 1 \\
\hline ESTADO CIVIL & 1 & 1 & 2 & 2 & 2 & 2 & 3 & 3 & 3 \\
\hline CERTIF. MINUSVALÍA & 1 & 1 & 0 & 0 & 0 & 0 & 0 & 0 & 0 \\
\hline HORAS SEMANALES & 5 & 4 & 3 & 3 & 3 & 4 & 3 & 6 & 4 \\
\hline SEXO & 0 & 1 & 0 & 0 & 0 & 0 & 0 & 0 & 0 \\
\hline \multicolumn{10}{|c|}{ DISCAPACIDADES: } \\
\hline DESPLAZARSE & 0 & 0 & 1 & 1 & 1 & 1 & 0 & 1 & 1 \\
\hline DEAMBULAR & 1 & 1 & 1 & 1 & 1 & 1 & 1 & 1 & 1 \\
\hline CUIDARSE & 1 & 1 & 0 & 0 & 0 & 1 & 0 & 1 & 1 \\
\hline TAREAS & 1 & 1 & 1 & 1 & 1 & 1 & 1 & 1 & 1 \\
\hline \multicolumn{10}{|c|}{ SEVERIDAD } \\
\hline DESPLAZARSE & 1 & 1 & 1 & 2 & 2 & 2 & 1 & 3 & 2 \\
\hline DEAMBULAR & 1 & 2 & 2 & 2 & 3 & 3 & 2 & 3 & 3 \\
\hline CUIDARSE & 2 & 1 & 1 & 1 & 1 & 2 & 1 & 3 & 2 \\
\hline TAREAS & 2 & 3 & 3 & 3 & 3 & 3 & 3 & 4 & 3 \\
\hline
\end{tabular}

Codificación de las variables:

EDAD: años

ABVD, CERTIF. MINUSVALÍA, DISCAPACIDADES: 1 presencia; 0 ausencia

ESTADO CIVIL: 1 soltero; 2 casado; 3 viudo

HORAS SEMANALES: 3 entre 15 y 30; 4 entre 31 y 40; 5 entre 41 y 60; 6 más de 60 horas

SEXO: 1 hombre; 0 mujer

SEVERIDADES: 1 sin dificultad; 2 dificultad moderada; 3 dificultad grave; 4 imposibilidad para realizar la actividad

Tabla 5

Descripción de la población integrante de cada categoría de dependencia

\begin{tabular}{|c|c|}
\hline GRUPO & CARACTERÍSTICAS \\
\hline $\begin{array}{c}1 \\
119.246 \\
\text { personas } \\
(8,6 \% \mathrm{~s} / \text { total })\end{array}$ & $\begin{array}{l}\text { Grupo más joven, igualdad varones-mujeres ( } 49 \% \text { ), tienen ABVD el } 77 \% \text { y certificado de minusvalía } \\
\text { el } 73 \% \text {, todos solteros, horas semanales de cuidado en media entre } 31 \text { y } 40 \text { recibiendo más de } 30 \text { horas } \\
\text { a la semana el } 69 \% \text {. } \\
\text { Respecto a las discapacidades es el grupo menos afectado: DESPLAZARSE el } 38 \% \text {, DEAMBULAR el } \\
54 \% \text {, CUIDARSE el } 64 \% \text { y TAREAS el } 66 \% \text {. }\end{array}$ \\
\hline $\begin{array}{c}2 \\
18.774 \\
(1,3 \%)\end{array}$ & $\begin{array}{l}\text { Segundo grupo más joven (de } 13 \text { a } 36 \text { años) y único grupo con más varones }(61 \%) \text {. Tienen ABVD el } \\
73 \% \text { y certificado de minusvalía el } 69 \% \text {. El } 88 \% \text { son solteros, reciben en media de } 31 \text { a } 40 \text { horas } \\
\text { semanales de cuidado y el } 62 \% \text { más de } 30 \text { horas. } \\
\text { DESPLAZARSE el } 34 \% \text {, DEAMBULAR el } 58 \% \text {, CUIDARSE el } 50 \% \text { siendo lo más destacable } \\
\text { TAREAS que afecta al } 90 \% \text {. }\end{array}$ \\
\hline
\end{tabular}


Tabla 5 (Cont.)

Descripción de la población integrante de cada categoría de dependencia

\begin{tabular}{|c|c|}
\hline GRUPO & CARACTERÍSTICAS \\
\hline $\begin{array}{c}3 \\
203.242 \\
(14,6 \%)\end{array}$ & $\begin{array}{l}\text { El } 62 \% \text { son mujeres, edades medias (de } 36 \text { a } 59 \text { ), el } 77 \% \text { tienen ABVD y certificado de minusvalía el } \\
44 \% \text { y el } 60 \% \text { están casados. Horas semanales en media de } 15 \text { a } 30 \text { recibiendo el } 41 \% \text { más de } 30 \text { horas. } \\
\text { DESPLAZARSE el } 50 \% \text {, DEAMBULAR el } 57 \% \text {, CUIDARSE el } 38 \% \text { y TAREAS el } 81 \% \text {. }\end{array}$ \\
\hline $\begin{array}{c}4 \\
322.516 \\
(23,1 \%)\end{array}$ & $\begin{array}{l}\text { Grupo con mayor proporción de personas casadas ( } 68 \% \text { ), el } 67 \% \text { son mujeres, edades medias-altas ( } 60 \text { - } \\
72 \text { años), tienen ABVD el } 83 \% \text { y certificado de minusvalía el } 25 \% \text {. Reciben horas semanales en media } \\
\text { de } 15 \text { a } 30 \text { y el } 42 \% \text { más de } 30 \text { horas. } \\
\text { DESPLAZARSE el } 54 \% \text {, DEAMBULAR el } 70 \% \text {, CUIDARSE el } 38 \% \text { y TAREAS el } 79 \% \text {. }\end{array}$ \\
\hline $\begin{array}{c}5 \\
198.170 \\
(14,2 \%)\end{array}$ & $\begin{array}{l}\text { Más mujeres }(71 \%) \text {, edades medias-altas }(73-77) \text {, el } 89 \% \text { tienen ABVD y certificado de minusvalía el } \\
15 \% \text {, el } 54 \% \text { están casados. Horas semanales de cuidado en media de } 15 \text { a } 30 \text { recibiendo el } 43 \% \text { más de } \\
30 \text { horas. } \\
\text { DESPLAZARSE el } 55 \% \text {, DEAMBULAR el } 76 \% \text {, CUIDARSE el } 42 \% \text { y TAREAS el } 81 \% \text {. }\end{array}$ \\
\hline $\begin{array}{c}6 \\
188.495 \\
(13,5 \%)\end{array}$ & $\begin{array}{l}\text { Más mujeres }(73 \%) \text {, edades medias-altas }(78-83) \text {, tienen ABVD el } 90 \% \text { y certificado de minusvalía el } \\
10 \% \text {, muchos }(48 \% \text { ) son viudos, horas semanales en media de } 15 \text { a } 30 \text { recibiendo el } 48 \% \text { más de } 30 \text {. } \\
\text { DESPLAZARSE el } 56 \% \text {, DEAMBULAR el } 79 \% \text {, CUIDARSE el } 53 \% \text { y TAREAS el } 86 \% \text {. }\end{array}$ \\
\hline $\begin{array}{c}7 \\
136.240 \\
(9,8 \%)\end{array}$ & $\begin{array}{l}\text { Más mujeres }(74 \%) \text {, edades altas }(82-90) \text {, el } 78 \% \text { tienen ABVD y sólo un } 4 \% \text { certificado de } \\
\text { minusvalía, más de } 2 / 3 \text { de las personas están viudas, horas semanales en media de } 15 \text { a } 30 \text { recibiendo el } \\
32 \% \text { más de } 30 \text { horas (mínimo registrado en los nueve grupos). Grupo menos afectado en términos de } \\
\text { severidad y horas de cuidado. } \\
\text { DESPLAZARSE el } 26 \% \text {, DEAMBULAR el } 65 \% \text {, CUIDARSE el } 25 \% \text { y TAREAS el } 85 \% \text {. }\end{array}$ \\
\hline $\begin{array}{c}8 \\
121.297 \\
(8,7 \%)\end{array}$ & $\begin{array}{l}\text { Grupo con más mujeres (el } 76 \% \text { ) y más número de horas semanales de cuidado (en media de } 41 \text { a } 60 \\
\text { recibiendo el } 38 \% \text { más de } 60 \text { horas). Edades altas ( } 82-90 \text { ), todos tienen ABVD y certificado de } \\
\text { minusvalía el } 10 \% \text {. Están viudos el } 62 \% \text {. Grupo más afectado por la dependencia en términos de } \\
\text { prevalencia, severidad y horas de cuidado. } \\
\text { DESPLAZARSE el } 85 \% \text {, DEAMBULAR el } 98 \% \text {, CUIDARSE el } 94 \% \text { y TAREAS el } 95 \% \text {. }\end{array}$ \\
\hline $\begin{array}{c}9 \\
86.941 \\
(6,2 \%)\end{array}$ & $\begin{array}{l}\text { Grupo más longevo (de } 90 \text { a } 99 \text { años), mujeres el } 74 \% \text {, el } 94 \% \text { tienen ABVD y certificado de } \\
\text { minusvalía el } 5 \% \text {. Predominan las personas viudas (79\%). Horas semanales que reciben de cuidados en } \\
\text { media de } 31 \text { a } 40 \text { recibiendo y el } 29 \% \text { más de } 40 \text {. Segundo grupo más afectado. } \\
\text { DESPLAZARSE el } 62 \% \text {, DEAMBULAR el } 87 \% \text {, CUIDARSE el } 75 \% \text { y TAREAS el } 92 \% \text {. }\end{array}$ \\
\hline
\end{tabular}

Existen más mujeres (67\%) que hombres, con edades comprendidas entre los 6 y los 99 años con una media de 68 años, el $85 \%$ tienen ABVD y el $24 \%$ poseen certificado de minusvalía. El 44\% están casados. Respecto a las horas semanales de cuidado, el $49 \%$ está en valores entre 4 y 6 (más de 31 horas). En relación al porcentaje de población dependiente con niveles elevados de severidad-los máximos de la escala del INE, nive- les 3 y 4- para las discapacidades de desplazarse, deambular sin medio de transporte, cuidarse y realizar tareas son $52 \%, 72 \%$, $48 \%$ y $68 \%$, respectivamente. En la tabla 5 se detallan las características de cada uno de los nueve grupos o categorías de dependencia diferenciadas estadísticamente.

El número medio de horas semanales de cuidados en todos los casos está situado 
Tabla 6

Impacto de las discapacidades y severidades por grupos (\% población afectada)

\begin{tabular}{|l|c|c|c|c|c|c|c|c|c|}
\hline & \multicolumn{7}{|c|}{ CONGLOMERADO } \\
\cline { 2 - 10 } & $\mathbf{1}$ & $\mathbf{2}$ & $\mathbf{3}$ & $\mathbf{4}$ & $\mathbf{5}$ & $\mathbf{6}$ & $\mathbf{7}$ & $\mathbf{8}$ & $\mathbf{9}$ \\
\hline desplazarse & $38 \%$ & $34 \%$ & $50 \%$ & $54 \%$ & $55 \%$ & $56 \%$ & $26 \%$ & $85 \%$ & $62 \%$ \\
\hline severidad 3-4 & $23 \%$ & $20 \%$ & $28 \%$ & $32 \%$ & $32 \%$ & $37 \%$ & $7 \%$ & $70 \%$ & $44 \%$ \\
\hline deambular & $54 \%$ & $58 \%$ & $57 \%$ & $70 \%$ & $76 \%$ & $79 \%$ & $65 \%$ & $98 \%$ & $87 \%$ \\
\hline severidad 3-4 & $33 \%$ & $34 \%$ & $33 \%$ & $44 \%$ & $51 \%$ & $56 \%$ & $33 \%$ & $87 \%$ & $65 \%$ \\
\hline cuidarse & $64 \%$ & $50 \%$ & $38 \%$ & $38 \%$ & $42 \%$ & $53 \%$ & $25 \%$ & $94 \%$ & $75 \%$ \\
\hline severidad 3-4 & $39 \%$ & $30 \%$ & $22 \%$ & $25 \%$ & $29 \%$ & $36 \%$ & $10 \%$ & $78 \%$ & $55 \%$ \\
\hline realizar tareas & $66 \%$ & $90 \%$ & $81 \%$ & $79 \%$ & $81 \%$ & $86 \%$ & $85 \%$ & $95 \%$ & $92 \%$ \\
\hline severidad 3-4 & $56 \%$ & $77 \%$ & $62 \%$ & $62 \%$ & $65 \%$ & $70 \%$ & $64 \%$ & $90 \%$ & $81 \%$ \\
\hline más de 30 horas & $69 \%$ & $62 \%$ & $41 \%$ & $42 \%$ & $43 \%$ & $48 \%$ & $32 \%$ & $82 \%$ & $67 \%$ \\
\hline
\end{tabular}

como mínimo entre las 15 y las 30 horas, agravándose en los casos de los grupos 8 -edades medias superiores a los 86 años- y 1 -edad media de 9 años-. Los porcentajes de población afectada por cada grupo de discapacidad, junto con los máximos grados de severidad y horas de cuidados en la tabla 6 .

\section{DISCUSIÓN}

Como consecuencia del análisis realizado hay que destacar dos resultados. En primer lugar, que la situación de dependencia no es propia ni exclusiva de las personas mayores de 64 años, tal y como evidencian los grupos del 1 al 4 . Es más, el grupo 4 engloba a personas con edades entre los 60 y los 72 años. En segundo lugar, se constata la existencia de un perfil evolutivo de la dependencia en la población de más de 60 años -grupos 4 a
9-, destacándose la situación peculiar de las personas de 82 a 90 años, ya que con el mismo perfil sociodemográfico -mujeres, con edades entre 82 y 90 años- se registran dos realidades opuestas en términos de situación de dependencia: un grupo refleja el máximo nivel de severidad y el otro el mínimo de todos los registrados.

Los perfiles obtenidos a partir de las categorías anteriores permiten conocer con mayor profundidad la heterogénea realidad existente entre las personas en situación de dependencia. El perfil tipo se caracteriza por la presencia de discapacidades asociadas a las ABVD y por una mayor incidencia en mujeres que en hombres en todos los grupos identificados, a excepción del número 2 -varones jóvenes-. La severidad se va agravando con la edad, hecho verificado en todos los grupos excepto en el número 7 . 
Cada una de las nueve categorías representa un perfil de dependencia distinto, no sólo en términos de discapacidades asociadas a las AVD sino también en términos de severidad, utilizando tanto el criterio del INE como el alternativo ${ }^{24}$. Los grupos reflejan una evolución de una situación de dependencia que lleva asociado un agravamiento a medida que aumenta la edad, con excepción de los grupos 1 y 2 de jóvenes. Hay que destacar los grupos 7 y 8, que teniendo igual perfil en edad y sexo, presentan dos realidades opuestas, con lo que, creemos que puede no ser correcto afirmar rotundamente que por sí solos, el sexo y la edad determinan situaciones de dependencia.

Respecto a la severidad, se confirman parte de los resultados obtenidos por Alegre et $\mathrm{al}^{24}$ relativos a las diferencias de valoración de la severidad al utilizar el criterio del INE y el alternativo. Así, se aprecia que el criterio del INE infravalora el grado de dependencia de los grupos 3 a 6 , que coincide que tienen menores tasas de severidad absolutas en los 4 grupos de discapacidades asociadas a las AVD.

Los resultados obtenidos no pueden ser comparados directamente con los de otras investigaciones $^{7-11}$ que diferencian situaciones pero centrándose en características o aspectos asociados al uso de servicios sanitarios por parte sólo de personas mayores, con información sobre el estado de salud percibido, ayudas recibidas, raza, nivel de estudios, antecedentes familiares, etc... Existen estudios ${ }^{11}$ que incluyen a las AVD pero sólo el número sin diferenciar de qué actividad se trata ni su severidad.

Como continuidad al trabajo presentado se profundizará en el análisis de los grupos 7 y 8 para determinar los factores que inciden en éstas situaciones. Asimismo, se estudiará el impacto de las codiscapacidades en cada una de las categorías para, en una fase posterior, trabajar en la elaboración de un índice (como sugieren otros autores ${ }^{24}$ ) que cuantifi- que el impacto de todas las AVD y estudiar el efecto económico y social que puedan tener. De esta forma, se ofrecería, tal y como establece Puga ${ }^{1}$, una mejor respuesta a las necesidades generadas a consecuencia de la situación de dependencia.

\section{BIBLIOGRAFÍA}

1. Puga González D. La dependencia de las personas con discapacidad: entre lo sanitario y lo social, entre lo privado y lo público. Rev Esp Salud Pública $2005 ; 79: 327-30$.

2. Puga MD, Abellán A. El proceso de discapacidad. Un análisis de la Encuesta de Discapacidades, Deficiencias y Estado de Salud. Madrid: Fundación Pfizer; 2004.

3. Rodríguez Cabrero G. (coord.). La protección social de la dependencia. Madrid: IMSERSO; 1999.

4. European Commission. Social Protection for Dependency in Old Age in the 15 EU Members States and Norway. Luxembourg: Publications of the European Communities; 1999.

5. Wilkin D y Thompson D. User's Guide to Dependency Measures for Elderly People. Sheffield: University of Sheffield Joint Unit for Social Services Research; 1989.

6. Consejo de Europa. Políticas Sociales Innovadoras en la Ciudad, Conferencia de Oslo 22-24 Junio 2000, Volumen 1 y 2. Estrasburgo; 2000.

7. Walsh J. Trends and Characteristics of Disability Support Pensioners. Paper presented at National Forum on Future Directions for Disability Policy, Department of Social Security, 24-25 July 1997. Canberra; 1997.

8. Manton KG. The Characteristics of the Elderly LTC Population and its Service Use. Durham (NC): U.S. Department of Health and Human Services, Assistant Secretary for Planning and Evaluation Grant no. 87ASPE185A; 1991.

9. Manton KG y Liu K. Recent Changes in Service Use Patterns of Disabled Medicare Beneficiaries. Health Care Financ Rev. 1990 Spring;11(3): 51-66.

10. Lea RD, Etheredge GD, Freeman JN y Lloyd WB. Familial Disability Patterns in Individuals with 
Chronic Work-related Spine Injury/illness. Spine 2003; 28(19): 2292-2297.

11. Zunzunegui MV y Béland F. La salud de las personas mayores de Leganés. Rev Gerontol 1995; 5: 245-8.

12. Katz S, Ford AB, Moskowitz AW, Jackson BA, Jaffe MW. Studies of Illness in the Aged. The Index of ADL: A Standardized Measure of Biological and Psychosocial Function. JAMA 1963; 185: 914919.

13. Lawton MP, Brody EM. Assessment of Older People: Self-maintaining and Instrumental Activities of Daily Living. Gerontologist 1969; 9: 179-86.

14. Casado D y López G. Vejez, dependencia y cuidados de larga duración. Situación actual y perspectivas de futuro. Colección Estudios Sociales n ${ }^{\circ} 6$. Barcelona: Fundación La Caixa; 2001.

15. Ruigómez A y Alonso J. Validez de la medida de capacidad funcional a través de las actividades básicas de la vida diaria en la población anciana. Rev Gerontol 1996; 6: 215-223.

16. Martínez de la Iglesia J. et al. Valoración funcional de personas mayores de 60 años que viven en una comunidad urbana. Proyecto ANCO. Aten Primaria $1997 ; 20: 475-84$.
17. Eiroa Patiño P. et al. Discapacidades y necesidades de servicios en las personas mayores detectadas en la encuesta de salud OARS-Vigo. Med Clin (Barc) 1996; 106: 641-8.

18. Organización Mundial de la Salud. Clasificación Internacional de Deficiencias, Discapacidades y Minusvalías (CIDDM). Madrid: Inserso; 1997.

19. Puga González D. Dependencia y necesidades asistenciales de los mayores en España, una previsión a 2010. Madrid: Fundación Pfizer; 2001.

20. INE. Encuesta de Discapacidades, Deficiencias y Estado de Salud 1999: Metodología. Madrid: INE; 2001.

21. Mahoney FI, Barthel DW. Functional Evaluation: the Barthel Index. Md State Med J 1965; 14: 61-65.

22. Juez Martel P. Herramientas estadísticas para la investigación en Medicina y Economía de la Salud. Madrid: Centro de Estudios Ramón Areces; 2001.

23. INE. Resultados de la Encuesta de Discapacidades, Deficiencias y Estado de Salud. Madrid: INE; 2001.

24. Alegre A, Ayuso M, Guillén M, Monteverde M y Pociello E. Tasa de dependencia de la población española no institucionalizada y criterios de valoración. Rev Esp Salud Pública 2005; 79: 351-3. 